\title{
Research on the use of virtual reality in intervention and rescue activities
}

\author{
Alin Irimia ${ }^{1 *}$,Emilian Ghicioi ${ }^{1}$, Daniel Pupăzan ${ }^{1}$, and Cristian Nicolescu ${ }^{1}$ \\ ${ }^{1}$ National Institute for Research and Development in Mine Safety and Protection to Explosion - \\ INSEMEX Petroşani, Romania
}

\begin{abstract}
In recent years in the virtual reality the developers succeeded in creating an artificial environment that simulates reality so that the user has the impression of an almost real physical presence in the virtual world. By overlapping and synchronizing the projected images with spatial sounds and even forces that interact with the user, there is a detachment of the user from the real environment and an all-encompassing involvement in the virtual world. The virtual reality is present in aviation, medicine, military training and other top fields. The advantages of using the virtual environment are obvious for areas where inexperience can cause very high loss of life and material loss. Although the participants are immersed in the scenario of the virtual environment, the errors produced allow an evaluation of the mistakes made and their correction, the experience gained being then used in professional activity in the real world. Through the experience of the virtual environment, the fear of various dangers can be overcome, so that the objectives of the exercise can be fulfilled. The aim of the paper is to offer to the rescuers opportunities to interact with the elements of the virtual space to fulfill the purpose of the exercise.
\end{abstract}

\section{Introduction}

Virtual reality has entered our lives with the development of three-dimensional effects in the field of cinema. Using special 3D glasses and sound effects, the viewer is subjected to an immersive experience through which he will feel like an active participant in the movie's action. Technological development has allowed the emergence of new, more complex equipment, able to reproduce three-dimensional large buildings, both indoors and outdoors, and by using virtual reality the participant is able to take a virtual tour that will provide a similar experience with physical participation. By using special glasses and controllers that users hold in their hands, to control certain actions such as movement, a route is taken that can be a copy of an existing physical environment or a digitally created one. Both variants create a feeling of physical presence for the user, within the world perceived through glasses, and a detaching from the real environment. There are situations in which the scenario in which one or more users participate simultaneously, does not involve a separation of the virtual environment from the real one but an overlap, the environment thus obtained being

* Corresponding author: alin.irimia@,insemex.ro 
called augmented reality. The notion of virtual reality is somewhat complementary to that of augmented reality, the latter being rather an extension of reality, it does not replace reality with a virtual environment but changes the perception of reality over which virtual objects overlap. VR technology can be adapted to many areas, from games that offer participants a very realistic development, to construction of flight simulators that offer training possibilities by replicating the functionality of pilot cabins and subjecting pilots to extreme situations.

Intervention and rescue activities in toxic, explosive or flammable environments, involves exposing rescuers to dangers that may be partially or completely unknown to them, exercises performed with mannequins or people as victims not inducing emotion as intense as a virtual scenario. During a classic exercise, the rescuer may be concerned with correct execution of intervention and rescue procedures, intervention time or score obtained, not being exposed to situations inducing fear for his own safety and that of other members of the rescue team. Repeated exercises can increase the ability of rescue teams to rigorously follow a procedure, but the action takes place without an emotional stress and without being able to trigger feelings specific to real events.

\section{Components of a VR system}

In order to be successful in achieving the goal of subjecting rescuers to experiences similar to reality with the help of virtual reality and augmented reality, a number of key elements are needed:

\subsection{Three-dimensional work environment}

Rescuers participating to rescue or intervention in virtual environments will have to travel a route, from a virtual training ground or a virtual space, that reproduces part of a technological platform, on which an event took place resulting in the occurrence of toxic gases, fires or explosions. This virtual environment, in which rescuers intervene, can be affected by different scenarios that the coordinator has at his disposal, to test the team's reaction. This complex scenario is attained with the help of a software platform in which three-dimensional footage of some industrial sections can be inputted, for example an installation in a refinery. If in a classic shooting of that installation we will see the image only from camera perspective, in the virtual space you can enter, move and interact with various virtual objects or even feel the effort to move them. This type of interaction with the work environment is obtained with the help of stereoscopic vision.

\subsection{VR headset}

The stereoscopic image is achieved with the help of glasses or headsets (fig.1) that the user wears on his head. 


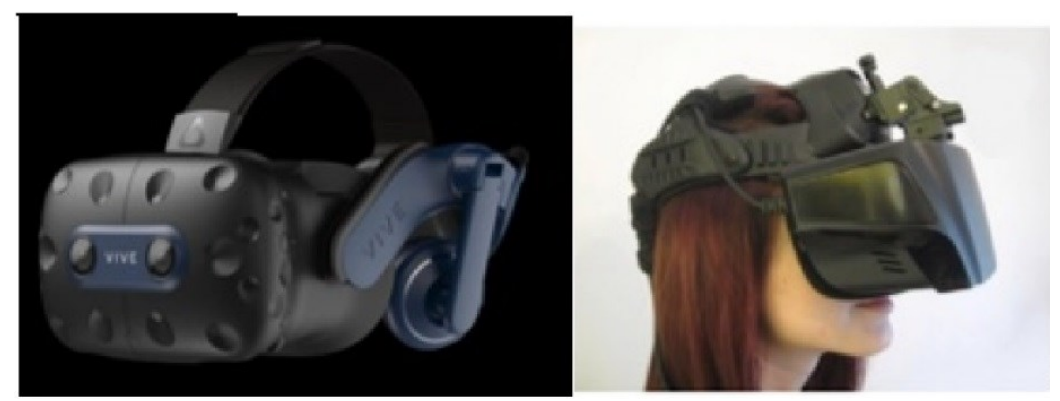

Fig. 1. VR headsets

It contains two displays, one for each eye, that will receive different images from the computer running the script, as perspectives to be transmitted to the eyes. Having a different image received by each eye correlated with body movement, stereoscopic images are created with the help of sensors placed near the eyes. The two displays can be high resolution, the viewing angle even reaching 120 degrees. The stereo audio system is also fitted on the headset and will independently play sounds pertain to right and left ear. In terms of functionality, the headphones can be stand-alone or wired to connect to peripheral devices.

\subsection{Gloves with sensors}

By using these gloves, equipped with a network of sensors able of detecting shape and movement of hands (fig. 2), interaction with objects is determined and object's position in the visual field is permanently adjusted. The gloves contain magnetic sensors to measure distance between fingers, accelerometers, gyroscopes and motion sensors to perceive movement and orientation of hands. Data transfer to external devices is done via USB cable or Bluetooth connection.

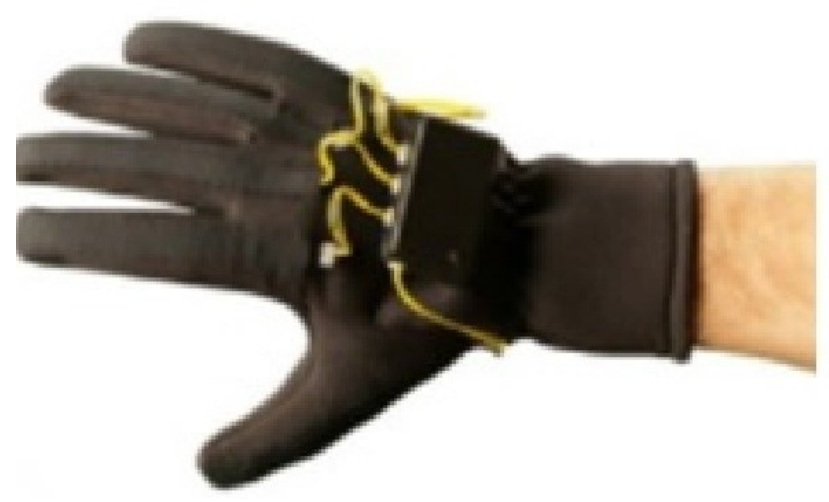

Fig. 2. VR glove

When it is necessary to transmit feedback related to the moved object, gloves equipped with vibro-tactile actuators and haptic sensors are used (fig. 3). With the help of these gloves, one can simulate interaction with objects and a hand-like sensation similar to real objects. 


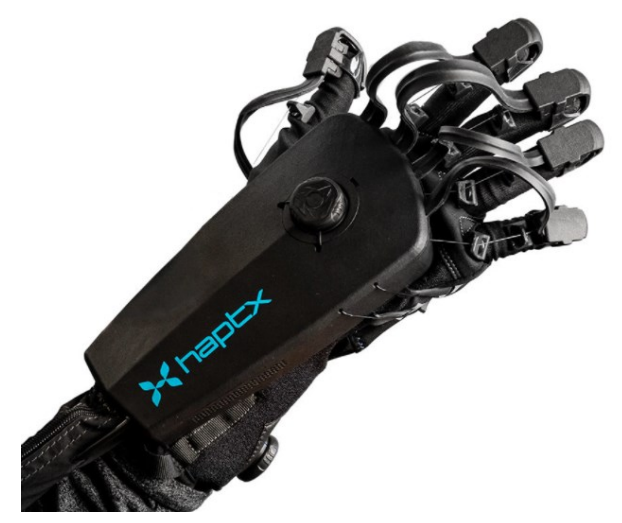

Fig. 3. VR glove with haptic sensors

\subsection{Motion sensors}

They are intended for tracking or predicting body, head or foot movement, depending on scenario specifics, used in the training process. Sensors allow the detection of 360-degree motion. In terms of technology used, sensors can be magnetic, inertial (fig. 4) or optical.

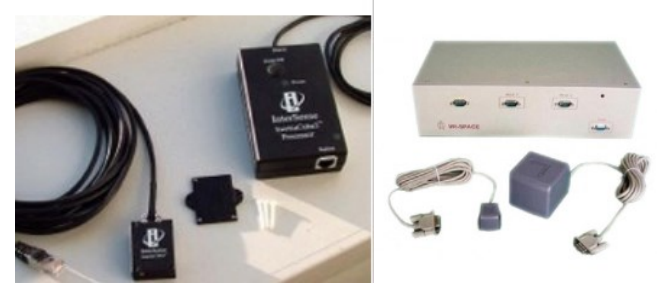

Fig. 4. Inertial and magnetic sensors

To follow head or body parts movements it is sufficient to use 2-4 magnetic or inertial sensors and to track movement of the whole body, optical sensors and receivers are more useful (Fig. $5)$.

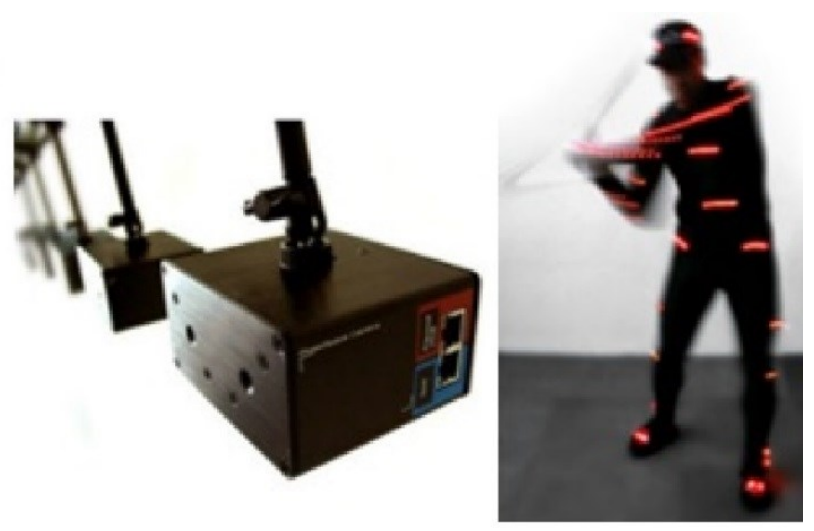

Fig. 5. Optical sensors and signal reception system 


\subsection{D Controller}

It is used for interacting and transmitting various pre-set commands to the virtual environment, by using the buttons that the controller is equipped with. It can perform functions of changing the viewing angle, moving objects on the 3 axes. For a more realistic perception, some controllers allow an in-stages setting of the user-programmable push-button resistance. In the training process for a technological intervention, depending on activity field, it may be necessary to perform a large number of operations, thus the need for a large number of buttons, but also the need to bring hands in the user's field of vision to see both hands movement and the result of the action on the controller.

Some controllers allow visualization of hand movements and may have functionality similar to VR gloves, being equipped with haptic sensors (fig. 6). The correlation between pressure exerted by fingers on buttons, 3D interpretation of complex hand movements and changing the viewing angle according to these actions is very important to provide a natural perception of operations performed by the user.
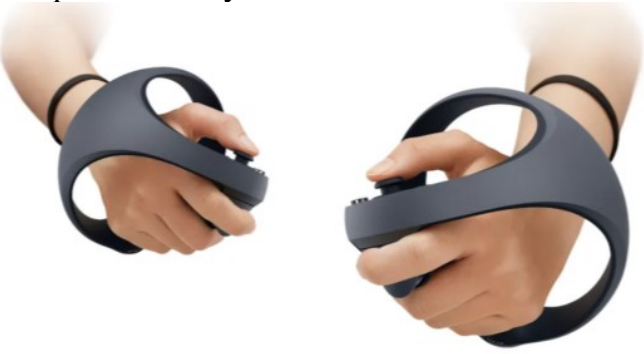

Fig. 6. Controller equipped with haptic sensors

Rescue activities in industrial environments posing a risk of explosive / flammable or toxic gases, both on surface but especially underground, are difficult because of major risk of fires or explosions in a very short time. Even testing of insulating devices can be implemented in the virtual space, team members participating in the virtual exercise individually checking the insulating devices in terms of tightness and triggering of the audible warning device. Team interaction, both at software and hardware level, is necessary for the use of virtual reality in the process of training rescuers, the minimum number of rescuers that can intervene being at least 3. [1] In order to maintain practical training at high levels, rescuers need to periodically perform simulations of technological interventions in all installations of the industrial objective posing a danger of toxic, explosive or flammable gases occurrence.

The success of a real emergency intervention depends on rescuers' degree of familiarity with equipment and technological processes in areas where damages occur and with technological processes necessary to limit and then eliminate it. [2] Because many industrial activities posing danger of toxic, explosive or flammable gases are carried out continuously, it is difficult to carry out rescue training in which to perform specific technological operations of isolating the damaged area, in order to eliminate the damage.

By using VR equipment, the training process can be carried out without interfering with the production process and, in case technical changes are made to the technological flow, they can be implemented in the virtual space that reproduces the respective section. By using the virtual reality equipment in the training process, rescuers detach themselves from the real world, immerse themselves in the virtual space, images in the VR headset becoming very real during training. [3] Compared to an exercise carried out in a training facility, where only the ability to work with the isolating device and the resistance to effort can be tested, in the virtual space, through the rescue intervention scenario, rescuers can also face feelings of fear and mental stress caused by images and realistic sounds they face, the emotional involvement 
being much stronger. The concept of virtual reality has not reached its maximum development potential, the equipment used is in a continuous process of development through increasingly high-performance sensors that can react to the finest body movements, to increasing processing capacity and growing video resolutions. [4] By simulating interventions with complex scenarios, team safety can be increased, as the rescue activity is generally subject to risks that cannot be fully foreseen, especially in the case of rescuers in underground mining industry[5] .

VR technology has been well implemented in many industrial fields but probably none of them have found so many challenges related to the need to perform many body movements in a small volume. Characteristic of many limited spaces, such as technological interventions for certain large machines is the lack of oxygen. In these cases it is imperative to use insulating protective devices and rescuers. The use of VR equipment in the training of the intervention and rescue teams can lead to states of dizziness, discomfort, especially for working in a limited space. Compared to other types of activities, the intervention and rescue is an activity exclusively for teams. During the intervention, the rescuers are in constant contact, both during the displacement and when performing the actual technological intervention activities. A VR system dedicated to the rescue activity must offer the possibility of simultaneous use for at least three team members, thus training the staff to work in a team and to increase the safety of the team according to the first principle of rescue.

Rescuers' training can be taken to a higher level by using virtual reality, that must be seen as a complementary method, conventional methods, as training in the training facility with the use of isolating devices, being also necessary. In the virtual space, theoretical knowledge of rescuers can be verified by creating scenarios for the development of dangerous events and the way in which the team intervenes. The rescue team is equipped with a VR headset and related controllers, enters the virtual environment where it receives information about the event that took place. Through interaction between team members, the list of needed intervention equipment is established, the isolating devices are checked and then the team leader orders the start of intervention according to the scenario. Actions of each member of the rescue team can be monitored, marked and then evaluated at the end of the exercise in order to improve intervention. Personnel's growth is ensured by the fact that the infrastructure needed to create the virtual environment consists of a modular hardware, that can be permanently improved by adding sensors or replacing them with more efficient ones and a software that can be permanently upgraded.

\section{Conclusions}

VR technology allows subjects' immersion in a virtual world, detaching from the real environment, images and sounds perceived in the headset becoming the new reality.

The virtual space is suitable for rescuers' training, being a close replica of the work environment; during virtual training rescuers can interact with facilities and equipment similar to those used in real spaces, to limit the spread of a dangerous event and to eliminate damage.

For training in a virtual environment, the VR equipment and the intervention scenario must be adapted to teamwork to allow members to interact. During an exercise in the virtual space, members of rescue teams work together, training having the role of increasing intervention capacity of the whole team, not just one member.

Present technical means, complex types of equipment and sensors allow configurations to be adapted to many industrial activities and specific intervention scenarios to be elaborated; permanent development of hardware components needed for creating the virtual space will allow, in the future, a wider spread of virtual training. 
The virtual space is ideal for performing complex exercises in real production areas, especially for industrial activities posing danger of toxic, explosive or flammable gases that are carried out continuously, where it is difficult to carry out rescue training in which to perform specific technological operations of isolating the damaged area, in order to eliminate the damage.

Training in the virtual space isolates rescuers from the real space and through images and sounds received in the VR headset, the state of fear and awareness of danger, specific to a real intervention, can be induced, thus psychologically preparing the team for a real situation.

Virtual reality technology is a complementary method to more classic methods of rescuers' training and cannot replace but can improve them and it can increase the degree of safety for intervention and rescue teams.

\section{References}

1. Gaman, A. G. Principles, techniques and procedures of intervention and rescue in toxic / explosive / flammable environments, Insemex Publishing House, Petrosani, 2007.

2. Gaman, A. G., Pupazan, D., Lupu, C. Procedures and regulations of the intervention and rescue formations in toxic / explosive / flammable environments, INSEMEX Publishing House, Petrosani, 2009.

3. Gaman, G. A., et al. Principles, techniques and procedures for intervention and rescue in toxic / explosive / flammable environments, Insemex Publishing House, Petrosani, 2007

4 Irimia, A et al. Using drones in support of rescue interventions teams in toxic/flammable/explosive environments, Environmental Engineering and Management Journal, Iasi, Vol 18. No. 4 831-837, 2019

5. Gaman, G. A., et al. Procedures and regulations of intervention and rescue teams in toxic / explosive / flammable environments, Insemex Publishing House, Petrosani, 2009

6. http://www.vive.com/us/, [Accessed 8.09.2021]

7.http://www.immersion.fr/en/products-services/solutions-3i/vr-in-a-case-by-immersion/, [Accessed 8.09.2021]

8. https://haptx.com/technology/, [Accessed 8.09.2021] 